\title{
Dispersion of longitudinal plasmons for a quasi-two-dimensional electron gas
}

\author{
W. H. Backes* \\ Technical University of Eindhoven, Department of Physics, P.O. Box 513, NL-5600 MB Eindhoven, The Netherlands \\ F. M. Peeters, F. Brosens, and J. T. Devreese ${ }^{\dagger}$ \\ University of Antwerp (UIA), Department of Physics, Universiteitsplein 1, B-2610 Antwerpen, Belgium
}

(Received 30 October 1991)

\begin{abstract}
Confinement of electrons in ultrathin metallic films leads to subbands. By increasing the thickness of the electron layer, the subbands will dissolve into a quasicontinuum, with the number of electrons per unit volume kept constant. Within the random-phase approximation, the two-dimensional plasmon, which originally follows Stern's dispersion relation, becomes a longitudinal surface plasmon. The plasmon excitations of a model metallic film are investigated by including all subbands. Single-particle excitations, which exhibit the depolarization shift, converge into the plasma excitation spectrum. With further increases in the film thickness, the bulk plasmon arises and the surface plasmon remains. Our analysis shows how quantum size effects evolve into hydrodynamical classical size effects with increasing thickness of the film.
\end{abstract}

\section{INTRODUCTION}

Molecular-beam epitaxy has recently been successful at growing ultrathin uniform metallic layers between two insulators and semiconducting substrates. ${ }^{1-6}$ These layered metal films possess no granular structures, unlike the evaporated or sputtered metal films which were used in the past. ${ }^{7}$ The thickness $(1.5-100 \mathrm{~nm})$ of the epitaxial metallic layers can be arranged to be on the order of the de Broglie wavelength $(0.5 \mathrm{~nm})$ of electrons in metals. The wide range of layer thicknesses makes it possible to study how quantum size effects (QSE) are transferred into well-studied classical size effects (CSE).

Plasma oscillations are an essential part of the dielectric properties of an electron gas. Plasmons in a threedimensional electron gas (3DEG) have been studied extensively. ${ }^{8}$ Of more recent interest is the research on plasmons in a quasi-two-dimensional electron gas (2DEG) of, e.g., semiconductor-insulator heterostructures. ${ }^{9,10}$ With epitaxial-growth technology, it becomes possible to study an intermediate electron gas which is between two and three dimensions. With increasing film thickness, the electron confinement disappears gradually and a bulk electron gas appears.

The confinement of electrons in the metallic layer leads to a discretization of energy levels and a subband structure in $\mathbf{k}$ space. Compared with the $2 \mathrm{DEG}$ in semiconductor heterostructures, the electron density in the epitaxial metal is very high. As a consequence, several subbands can be occupied. When wider metal layers are investigated, for a constant number of electrons per unit volume, the subbands shift together and more of them become occupied. In $\mathbf{k}$ space, a quasicontinuum will be built up and the 3DEG comes into existence. The aim of this paper is to investigate the transition of collective excitations for a $2 \mathrm{DEG}$ transferring into those of $3 \mathrm{DEG}$.

The band structure ${ }^{3}$ across the semiconductorinsulator-metal heterostructure produces geometrical quantization in the electronic layer. The relatively large band gap of the surrounding insulators and the absence of a band gap in the metal motivates the idea of confining the conducting electrons in a rectangular infinitely deep potential well. In Sec. II a simple model will be presented to study this geometrical confinement. We show the consequences of dissolving the discrete quantum states on the occupation of subbands in $\mathbf{k}$ space. Section III contains the dispersion relations for plasmons derived within the random-phase approximation (RPA). The lack of translation invariance forces us to use a nondiagonal dielectric matrix. We found that only when a few subbands are occupied are the depolarizing single-particle excitations ${ }^{9,11-15}$ and the two-dimensional plasmon recovered. ${ }^{16}$ Our approach is different from earlier investigations, in the sense that an almost unlimited number of subbands are included. This is the reason why we are able to recover the limit of the 3 DEG by a very thick layer. In Sec. IV we show how important intersubband plasmons are in comparison with intraband plasmons when wider metal layers are investigated. The theoretical approach is concerned with the energy loss of fast electrons. In the bulk limit, we found numerically a surface plasmon and a bulk plasmon, which is in agreement with hydrodynamical classical size effects. ${ }^{17}$ Our conclusions and discussions are given in Sec. V.

\section{MODEL OF A CONFINED ELECTRON GAS}

Both Schottky barriers are modeled by an infinitely deep potential well, with finite width $w$. The positively charged ions are treated in the jellium approach. The free-particle Hamiltonian possesses the eigenfunctions

$$
\psi_{\mathbf{k}, l}(\rho, z)=\frac{1}{\sqrt{A}} e^{i \mathbf{k} \cdot \rho} \zeta_{l}(z),
$$

where $A$ is the area of one metallic surface. In the $\rho=(x, y)$ plane these solutions are plane waves, where 
(a)

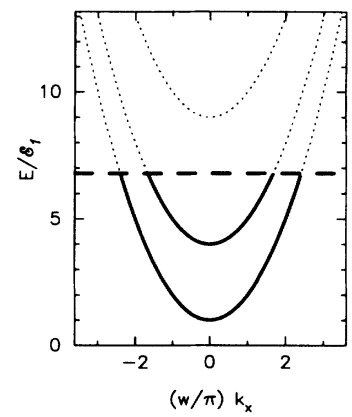

(b)

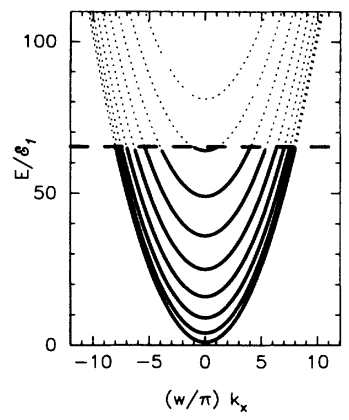

FIG. 1. The energy spectrum for two values of the subband occupation: (a) $l_{F}=2$ and (b) $l_{F}=8$. The Fermi levels are indicated by the horizontal dashed lines.

Born-von Kármán boundary conditions ${ }^{18,19}$ are used, to simulate an infinite system. In the $z$ direction the standing waves

$$
\zeta_{l}(z)=\sqrt{2 / w} \sin \left(\frac{l \pi z}{w}\right) \text { for } l=1,2, \ldots,
$$

are the wave functions, obeying Dirichlet boundary conditions. The two-dimensional wave vector $\mathbf{k}$ and the subband index $l$ constitute a suitable set of quantum wave numbers $(\mathbf{k}, \pi l / w)$, with the spin omitted. The eigenvalue spectrum is parabolic

$$
E_{\mathrm{k}, l}=\frac{\hbar^{2} k^{2}}{2 m}+\mathscr{E}_{l},
$$

with a subband offset of $\mathscr{E}_{l}=\hbar^{2} \pi^{2} l^{2} / 2 m w^{2}$. The essence of QSE is the direct incorporation of the layer thickness into the single-particle wave functions.

The occupation of the $(\mathbf{k}, \pi l / w)$ space is characterized by a stack of Fermi disks with different Fermi wave vectors $k_{F}(l)=(\pi / w) \sqrt{E_{F} / \mathscr{E}_{1}-1}$, where $E_{F}$ is the Fermi level. When gradually wider layers are investigated, assuming a constant number of electrons per volume unit $\left(n^{(3 D)}\right)$, more and more subbands become occupied (Fig. 1). The spacing between the Fermi disks becomes smaller (Fig. 2) and disks pile on top of each other to construct a Fermi sphere, with radius $k_{F}^{(3 D)}=\left(3 \pi^{2} n^{(3 D)}\right)^{1 / 3}$. In state space, as shown in Fig. 2, we have also indicated quan- (a)

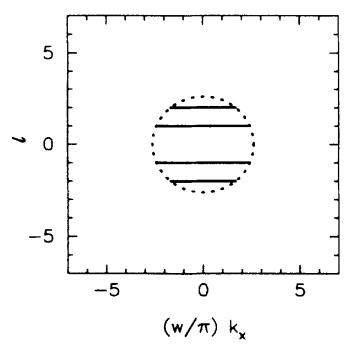

(b)

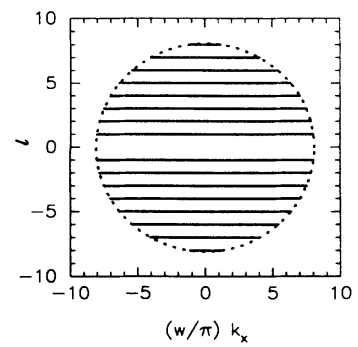

FIG. 2. The occupied states (solid lines) and the Fermi surface (dotted circle) for the situation in Fig. 1: (a) $l_{F}=2$ and (b) $l_{F}=8$. tum states with negative subband indices. This was done in order to obtain a Fermi sphere instead of the proper hemisphere. Dissolving the discrete quantum wave number $\pi l / w$ into a quasicontinuous wave-vector component $k_{z}$ leads to the transition of a quasi-2DEG into a 3DEG.

\section{DISPERSION OF PLASMONS}

Following Ehrenreich and Cohen, ${ }^{20}$ the density response to a perturbing external potential

$$
\boldsymbol{V}^{(\mathrm{ext})}(\boldsymbol{\rho}, z, t)=\boldsymbol{V}^{(\mathrm{ext})}(w, \mathbf{q} ; z) e^{i(\mathbf{q} \cdot \boldsymbol{\rho}-\omega t)}
$$

will be treated within the RPA approach, where we will include the discrete subband structure. ${ }^{21}$ This restriction to the longitudinal density-density response neglects the effects of retardation. ${ }^{22}$

The density perturbation gives the total potential

$$
\boldsymbol{V}=\boldsymbol{V}^{(\mathrm{ext})}+\boldsymbol{V}^{(s)}
$$

as a self-consistent scalar field. The screening potential $V^{(s)}$ is the solution

$$
\boldsymbol{V}^{(s)}(\omega, \mathbf{q} ; z)=\int_{0}^{\omega} d z^{\prime} \boldsymbol{V}^{(\boldsymbol{C})}\left(q ; z, z^{\prime}\right) \delta n\left(\omega, \mathbf{q}, z^{\prime}\right)
$$

of the Poisson equation connected to the induced electron density $\delta n$, with

$$
V^{(C)}\left(q ; z, z^{\prime}\right)=v_{q} e^{-q\left|z-z^{\prime}\right|}
$$

and the two-dimensional Fourier-Coulomb factor $v_{q}=2 \pi e^{2} / q$. The time-dependent single-particle Liouville equation relates, to first order, the density fluctuation to the total potential

$$
\delta n(\omega, \mathbf{q} ; z)=\int_{0}^{w} d z^{\prime} \Pi\left(\omega, q ; z, z^{\prime}\right) V\left(\omega, \mathbf{q} ; z^{\prime}\right) .
$$

The irreducible polarization $I I$ is given by

$$
\begin{aligned}
\Pi\left(\omega, q ; z, z^{\prime}\right)=\sum_{l=1}^{l_{F}} \sum_{l^{\prime}=1}^{\infty} & \Lambda_{l l^{\prime}}(\omega, q) \xi_{l}(z) \xi_{l^{\prime}}(z) \\
& \times \xi_{l}\left(z^{\prime}\right) \xi_{l^{\prime}}\left(z^{\prime}\right),
\end{aligned}
$$

where the integer part for $l_{F}=\operatorname{Int}\left(\sqrt{E_{F} / \mathscr{E}_{1}}\right)$ denotes the highest occupied subband and $\Lambda$ the polarizability function

$$
\begin{aligned}
\Lambda_{l l^{\prime}}(\omega, q)=\frac{2}{A} \sum_{\substack{\mathbf{k} \\
|\mathbf{k}|<k_{F}(l)}} & \frac{1}{\hbar \omega+E_{\mathbf{k}, l}-E_{\mathbf{k}+\mathbf{q}, l^{\prime}}} \\
& \left.-\frac{1}{\hbar \omega+E_{\mathbf{k}-\mathbf{q}, l^{\prime}}-E_{\mathbf{k}, l}}\right),
\end{aligned}
$$

where the extra factor 2 takes care of spin degeneracy. As usual a small positive imaginary part $i \gamma$ is included in the frequency $\omega$ to represent adiabatic switching of the Coulomb interaction. The polarizability matrix can be simplified to

$$
\begin{aligned}
\Lambda_{l l^{\prime}}(\omega, q)=\frac{q k_{F}(l)}{2 \pi E_{q}}\{ & K\left(u_{-}\left(\omega, q, l^{\prime}, l\right)\right) \\
& \left.-K\left(u_{+}\left(\omega, q, l^{\prime}, l\right)\right)\right\},
\end{aligned}
$$


where we introduced the definitions

$$
K(u)= \begin{cases}u-\left(u^{2}-1\right)^{1 / 2} & \text { if }|u|>1 \text { and } \operatorname{Re}(u)>0 \\ u+\left(u^{2}-1\right)^{1 / 2} & \text { if }|u|>1 \text { and } \operatorname{Re}(u)<0 \\ u-i\left(1-u^{2}\right)^{1 / 2} & \text { if }|u|<1\end{cases}
$$

and

$$
u_{ \pm}\left(\omega, q, l^{\prime}, l\right)=\frac{\hbar \omega \pm\left(E_{q}+\mathscr{E}_{l^{\prime}}-\mathscr{E}_{l}\right)}{\left(\hbar^{2} / m\right) q k_{F}(l)}
$$

The dielectric function $\kappa$ relates the external and total potentials as $V^{(e x t)}=\kappa V$. This yields the dielectric function inside the metal layer $\left(0 \leq z<+w, 0 \leq z^{\prime} \leq w\right)$

$$
\begin{aligned}
\kappa\left(\omega, q ; z, z^{\prime}\right)= & \delta\left(z-z^{\prime}\right) \\
& -\int_{0}^{w} d z^{\prime \prime} V^{(C)}\left(q ; z, z^{\prime \prime}\right) \Pi\left(\omega, q ; z^{\prime \prime}, z^{\prime}\right),
\end{aligned}
$$

and outside the sheet $\left(z<0, z^{\prime}<0\right.$ or $\left.z>w, z^{\prime}>w\right)$

$$
\kappa\left(\omega, q ; z, z^{\prime}\right)=\delta\left(z-z^{\prime}\right),
$$

while for other $\left(z, z^{\prime}\right)$ combinations we have $\kappa=0$.

Inside the metallic film the dispersion relation for the collective modes is determined by

$$
\operatorname{det}\left[\underline{1}-\underline{V}^{(C)}(q) \underline{\Pi}(\omega, q)\right]=0,
$$

where $\underline{1}, \underline{V}^{(C)}$, and $\underline{\Pi}$ are matrix representations of, respectively, the unit operator, the bare Coulomb potential, and the irreducible polarization in a complete set of eigenfunctions on the interval $0 \leq z \leq w$. The choice of the eigenfunctions is complicated by the fact that the effective Coulomb potential is a rather smooth function, whereas the polarization is strongly oscillatory. For numerical purposes it is of paramount importance to choose a suitable set of eigenfunctions such that only a relatively small number of matrix elements is important in the calculation of the determinant.

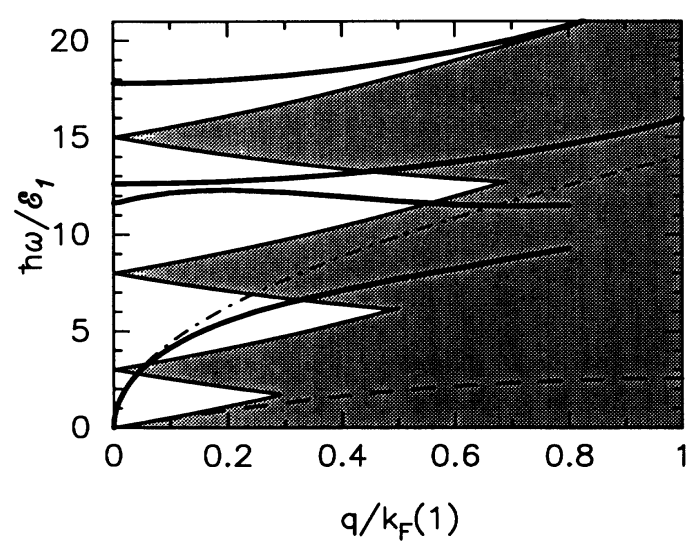

FIG. 3. The zero-point inventory of the dielectric determinant for one occupied subband (solid curves). The electronhole continuum is marked and shaded, also Stern's dispersion relation (dashed-dotted) is drawn; $E_{F} / \mathscr{E}_{1}=3.5, n^{(3 \mathrm{D})}=5 \times 10^{20}$ $\mathrm{cm}^{-3}, \gamma=0$.
Figure 3 contains an inventory of the zeros (thick solid curves) of the dispersion law for plasmons. The shaded area corresponds to the electron-hole continuum, where $\kappa$ and $\Lambda$ have imaginary parts. The existence of plasmons is disturbed in this region by single-particle excitations. Similar to Dahl and Sham, ${ }^{22}$ two types of zero-point branches are found. First there is the two-dimensional intraband plasmon (dashed-dotted curve) with frequency

$$
\omega^{(2 \mathrm{D})}(q)=\left(q 2 \pi e^{2} n^{(2 \mathrm{D})} / m\right)^{1 / 2},
$$

for $q \downarrow 0$ approaching Stern's dispersion relation, ${ }^{16}$ with $n^{(2 \mathrm{D})}=w n^{(3 \mathrm{D})}$. When the plasmon branch enters the shaded region, the collective oscillations will be damped and here the plasmon frequencies possess nonzero imaginary parts. For larger wave vectors the frequency tends to be lower than the corresponding Stern result. It should be emphasized that the RPA is only valid in the lowest order of $q$, see Ref. 23. Because of the present subband structure a mode-coupling effect is retained between intraband and interband collective modes, noted by Das Sarma in Ref. 14. Secondly, single-particle excitations are present, characterized by constant nonzero frequencies for $q=0$. The resonance frequencies

$$
\omega_{l l^{\prime}}^{2}=\left(\Delta_{l l^{\prime}} / \hbar\right)^{2}+\widetilde{\omega}_{p, l l^{\prime}}^{2}
$$

do not correspond to the subband splittings $\Delta_{l l^{\prime}}$ because of the depolarization effect. ${ }^{9,11,12}$ This effect is caused by Coulombic screening and leads to a positive shift of the transition energy above the subband separation: the depolarization shift. Figure 4 shows the position of the three lowest single-particle resonances as a function of the electron density. The depolarization shift is the distance of this curve from the dotted horizontal lines, which represent the subband splittings $\Delta_{l l^{\prime}}=\left(l^{2}-l^{\prime 2}\right) \mathscr{E}_{1}$. The dashed curve is obtained when we limit ourselves to one virtual subband $\left(l^{\prime}=1,2\right)$. It shows that the influence of higher virtual subbands on the depolarization shift is more pronounced with increasing electron density. Note

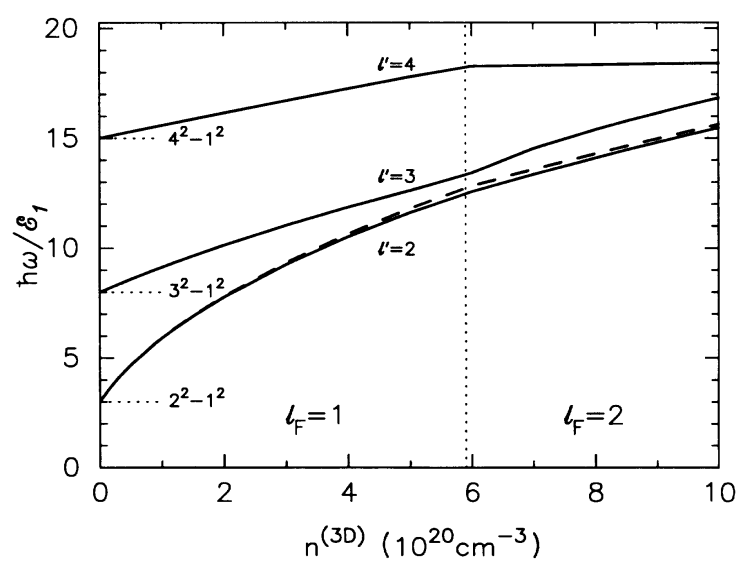

FIG. 4. The three lowest resonance frequencies for corresponding intersubband plasmons as a function of the electron density at constant layer thickness $w=2 \mathrm{~nm}$. For densities $n^{(3 \mathrm{D})}>5.9 \times 10^{20} \mathrm{~cm}^{-3}$ two subbands are occupied. The dashed curve is the result when only one unoccupied subband is included. 
that when the electron density approaches zero, the depolarization shift reduces to zero and the resonance frequencies correspond to the subband splittings. The frequency of the screening collective mode $\widetilde{\omega}_{p, l l^{\prime}}^{2}=\Omega_{p}^{2} f_{l l^{\prime}}$ is related to the oscillator strength $f_{l l^{\prime}}$ of an intersubband transition, which Chen, Chen, and Burstein ${ }^{11}$ pointed out. These oscillator strengths can only be obtained numerically, when several subbands are filled. Furthermore, we notice in Fig. 3 that two plasma branches are very close to each other, which occurs near the bulk plasma frequency $\Omega_{p}^{2}=4 \pi e^{2} n^{(3 \mathrm{D})} / m$. This is associated with the creation of a bulk plasmon, as will be seen in the next section.

\section{ENERGY LOSS OF FAST ELECTRONS}

The dielectric matrix is utilized to calculate the energy loss spectrum of fast electrons which enter the metal layer at normal incidence. The nonrelativistic velocity $v \gg \hbar k_{F}(1) / m$ of the external electron permits Born's approximation, treating this charge $e$ classically with potential

$$
V^{(\mathrm{ext})}(\mathbf{r}, t)=\frac{e}{|\mathbf{r}-\mathbf{v} t|}
$$

This perturbation leads to single particle and plasmon excitations inside the layer, and consequently to an energy and momentum loss of the external electron.

The induced density fluctuations exert a stopping force $-\nabla V^{(\text {ind })}$ to the electron, which yields an energy loss $d \mathcal{A}=e \mathbf{v} \cdot \nabla V^{(\text {ind })} d t$ per unit path length. The transformation

$V(\mathbf{r}, t)=\sum_{n} \int d^{2} q \int d \omega V_{n}(\omega, \mathbf{q}) e^{i(\mathbf{q} \cdot \rho-\omega t)} \widehat{e}_{n}(z)$

with the set $\hat{e}_{n}(z)=(1 / \sqrt{w}) e^{i 2 \pi n z / w}$ for $n=0, \pm 1, \ldots$, admits the use of the inverted dielectric matrix in $V^{(\text {ind })}=\left(\kappa^{-1}-1\right) V^{(\text {ext })}$. Only normal incidence of the external electron to the layer surface is considered. The energy loss is obtained as

$$
\mathcal{A}=\int d^{2} q \int_{0}^{\infty} d \omega \omega \mathcal{P}(\omega, q)
$$

where the absorption function $\mathcal{P}(\omega, q)$ indicates the probability for a transfer of energy $\hbar \omega$ and two-dimensional momentum $q$ to the electron gas

$$
\begin{aligned}
& \mathscr{P}(\omega, q)=\frac{e^{2}}{w \pi^{2}} \frac{1-\cos (\omega w / v)}{q^{2} v^{2}+\omega^{2}} \sum_{n n^{\prime}}\left.-\operatorname{Im} \kappa_{n n^{\prime}}^{-1}(\omega, q)\right] \\
& \times\left(\frac{1}{2 \pi n^{\prime} / w-\omega / v} \frac{1}{2 \pi n / w-\omega / v}+\frac{1}{2 \pi n^{\prime} / w+\omega / v} \frac{1}{2 \pi n / w+\omega / v}\right) .
\end{aligned}
$$

The absorption function is plotted in Figs. 5 for (a) one subband occupied and (b) two subbands occupied. The lowest resonance $\left(l^{\prime}=2\right)$ has a transfer probability which is about ten times larger than the next $\left(l^{\prime}=3\right)$ one. We found that is not necessary to include many virtual subbands when only the lowest subband is occupied. For example, when only the first two $\left(l^{\prime}=1,2\right)$ subbands are included, a qualitatively similar spectrum is obtained, except of course for the higher transitions $\left(l^{\prime}=3,4, \ldots,\right)$. We took for all spectra the charge velocity $v=\sqrt{1000 E_{F} / m}$, the density $n^{(3 \mathrm{D})}=5 \times 10^{20} \mathrm{~cm}^{-3}$ and the damping $\gamma=0.1 \Omega_{p}$, unless stated differently. As the width of the metal film increases, the lowest subband will be filled to a higher Fermi level, and the higher resonances shift towards the lower ones. As the occupation of higher subbands progresses, the two-dimensional plasma oscillation gains intensity, with respect to the singleparticle excitations [Fig. 5(b)]. This is shown more clearly in Fig. 6 where we plot the absorption spectrum as function of the frequency for a constant small wave vector $q=0.1 k_{F}^{(3 \mathrm{D})}$, for different values of the width of the layer. Notice that the two-dimensional plasmon evolves into a surface plasmon, with asymptotic frequency $\omega=\Omega_{p} / \sqrt{2}$. The position of the important peaks are plotted in Fig. 7 as function of the width of the layer. Towards this bulk limit also the three-dimensional plasmon arises (Fig. 7). The quantized electron motion perpendicular to the interfaces disappears in the spectrum. The three-dimensional plasma oscillations become more important, with respect to the surface oscillations.

The surface collective modes were investigated by Ritchie. ${ }^{17}$ Using the hydrodynamical CSE for a charged electron liquid, he found

$$
\omega(q)=\Omega_{p}\left(\frac{1-e^{-q w}}{2}\right)^{1 / 2},
$$

which is in agreement with the present numerical analysis in Fig. 8 (dashed curves), at least for small $q$ values. It is necessary to take a nonzero damping, otherwise $\operatorname{Im} \kappa=0$ outside the electron-hole continuum and no peak structure can be observed numerically for the plasmon branches. 

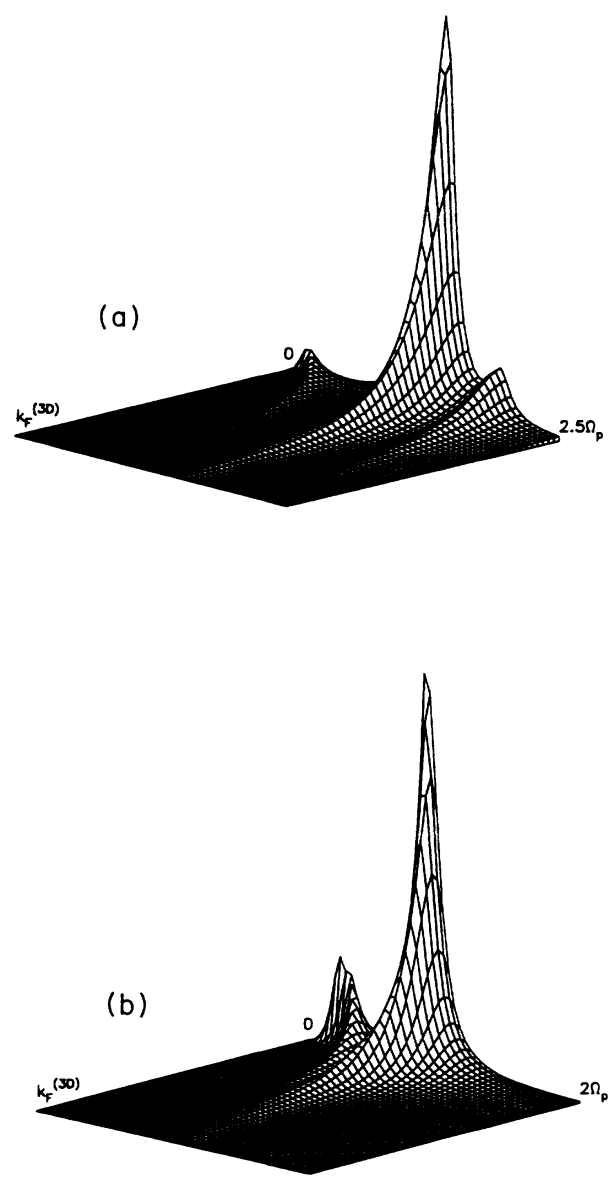

FIG. 5. The absorption function $\mathcal{P}(\omega, q)$ for (a) $l_{F}=1$, $E_{F} / \mathscr{E}_{1}=2.1$ and (b) $l_{F}=4, E_{F} / \mathscr{E}_{1}=17.6$. We used a damping of $\gamma=0.1 \Omega_{p}$.

\section{DISCUSSION AND CONCLUSIONS}

Geometrical confinement of electrons into a potential well creates a discrete subband structure. Because of the

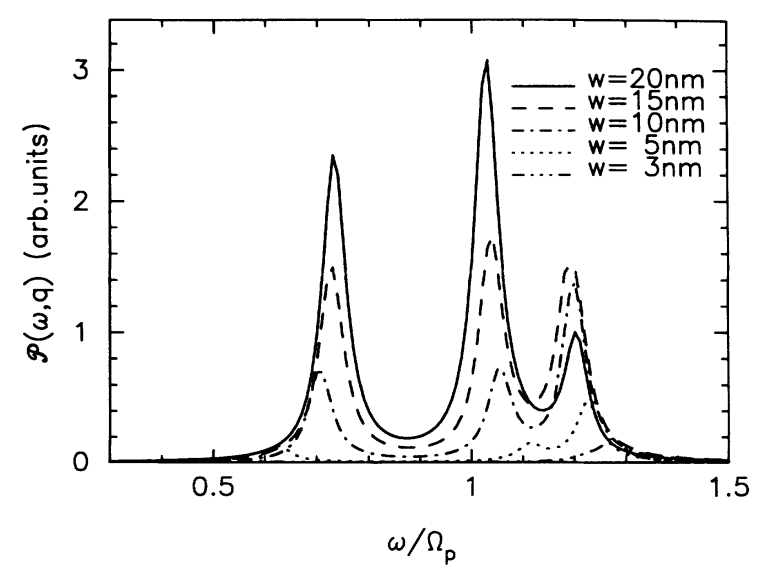

FIG. 6. The absorption function $\mathcal{P}(\omega, q)$ as function of the frequency for a constant $q=0.1 k_{F}^{(3 \mathrm{D})}$ and a damping of $\gamma=0.1 \Omega_{p}$ for different values of the layer width.

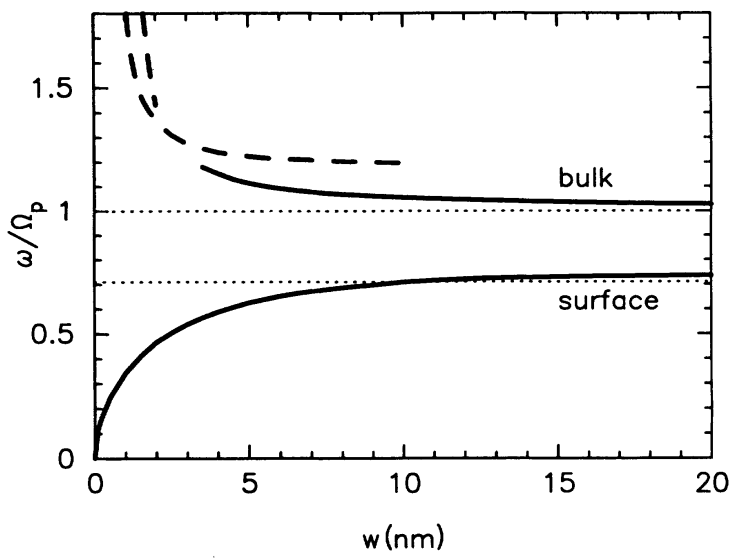

FIG. 7. The evolution of the plasmon dispersions at constant $q=0.1 k_{F}^{(3 \mathrm{D})}$ as a function of the layer thickness. We took $\gamma=0.01 \Gamma_{p}$.

relatively high electron density in a metallic layer, an (in principle) unlimited number of occupied subbands are present. This is in contrast to the quasi-2DEG in semiconductor-insulator heterostructures, where usually only one subband is occupied. For a fixed number of electrons per unit volume, the energy levels shift nearer to each other when wider metal layers are studied, and the correct three-dimensional limit for plasmon excitations is obtained. From the dispersion of the collective excitations as obtained from the nonlocal RPA dielectric matrix and the energy loss by fast electrons, we found (modified) two-dimensional plasma oscillations and depolarizing single-particle excitations. Coupling between intraband and intersubband plasmons causes a downward bending of the two-dimensional plasmon dispersion as compared with the strict two-dimensional result of Stern. In wider layers more subbands become occupied, the intersubband resonances approach each other, and the dispersion of the intraband plasmon changes into a longitudinal surface mode. The bulk plasmon appears as a plasma oscillation in three dimensions, and exceeds other collective modes in intensity. In the bulk limit the plasma oscillations agree with results from CSE.

The RPA dielectric description fails mainly for retardation, and exchange and correlation effects in semiconductor-insulator heterostructures. Retardation has been taken into account by solving the full set of Maxwell equations and it was found to be of minor importance. ${ }^{22}$ The major limitation of the RPA approach is the neglect of correlation and exchange for low electron densities. On the other hand, it is well known that the dynamic Hartree method is a successful technique to describe plasmons and single-particle excitations in the long-wavelength limit at metallic electron densities. Ando ${ }^{13,15}$ used a local correction term to take account of the excitonlike effect in heterostructures where a single subband is occupied, giving a further modification to the single-particle resonances. A more proper approach would be a dynamic Hartree-Fock calculation, as outlined in Ref. 24.

However, there is experimental evidence of QSE dis- 
(a)

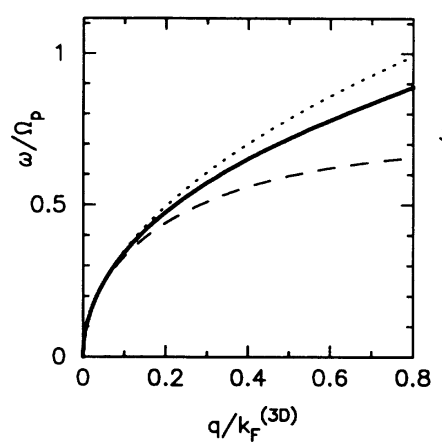

(b)

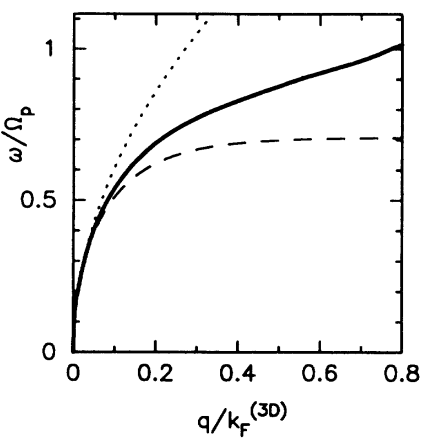

(c)

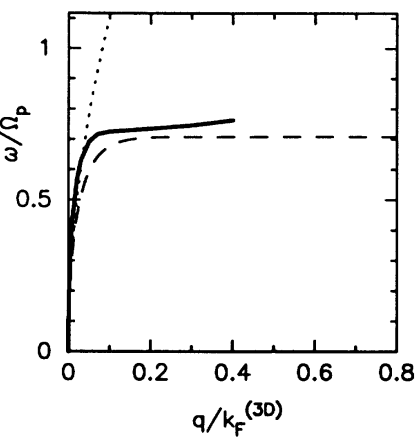

FIG. 8. The dispersion relation of the two-dimensional plasmon at (a) $w=1 \mathrm{~nm}$, (b) $w=3 \mathrm{~nm}$, and (c) $w=20 \mathrm{~nm} ; \gamma=0.01 \Omega_{p}$. The dispersion relations according to Stern and Ritchie are, respectively, given by the dotted and dashed curves.

solving into CSE in optical transmission and reflection experiments in sputtered gold films. ${ }^{25}$ Demonstrations of dissolving the discrete quantum states and the dispersion modification of the longitudinal plasmons in gradually wider metal layers are, except for energy-loss experiments, possible in infrared-absorption experiments, ${ }^{26,27}$ and eventually by means of a grating coupler. ${ }^{10}$

\section{ACKNOWLEDGMENTS}

Two of us (F.M.P. and F.B.) were supported by the Belgian National Science Foundation. This work was supported by FKFO (Fonds voor Kollektief Fundamenteel Onderzoek, Belgium), Project No. 2.0093.91 and IUAP-11.
*Also at Department of Physics, University of Antwerp, Antwerpen, Belgium.

${ }^{\dagger}$ Also at Technical University of Eindhoven and Institute for Applied Mathematics, Rijksuniversitair Centrum Antwerpen.

${ }^{1}$ J. Harbison, T. Sands, N. Tabatabaie, W. Chan, L. Florez, and V. Keramidas, Appl. Phys. Lett. 53, 1717 (1988).

${ }^{2}$ T. Sands, J. Harbison, N. Tabatabaie, W. Chan, H. Gilchrist, T. Cheeks, L. Florez, and V. Keramidas, Surf. Sci. 22, 1 (1989).

${ }^{3}$ N. Tabatabaie, T. Sands, J. Harbison, H. Gilchrist, and V. Keramidas, Appl. Phys. Lett. 53, 2528 (1988).

${ }^{4}$ S. Chambers, V. Loebs, and D. Doyle, J. Vac. Sci. Technol. B 8, 985 (1990).

${ }^{5}$ N. Tabatabaie, T. Sands, J. Harbison, H. Gilchrist, L. Florez, and V. Keramidas, Appl. Phys. Lett (to be published).

${ }^{6} \mathrm{M}$. Tanaka, H. Sakakibara, T. Nishinaga, N. Ikarashi, and K. Ishida (unpublished).

${ }^{7}$ H. Raether, Excitation of Plasmons and Interband Transitions by Electrons (Springer-Verlag, Berlin, 1980).

${ }^{8}$ D. Pines, Rev. Mod. Phys. 28, 184 (1956).

${ }^{9}$ T. Ando, A. Fowler, and F. Stern, Rev. Mod. Phys. 54, 437 (1982).

${ }^{10}$ D. Heitmann, Phys. Scr. T25, 294 (1989).

${ }^{11}$ W. Chen, Y. Chen, and E. Burnstein, Surf. Sci. 58, 263 (1976).
${ }^{12}$ S. Allen Jr., D. Tsui, and B. Vinter, Solid State Commun. 20, 425 (1976).

${ }^{13}$ T. Ando, Z. Phys. B 26263 (1977).

${ }^{14}$ S. Das Sarma, Phys. Rev. B 29, 2334 (1983).

${ }^{15}$ L. Wendler and V. Grigoryan, Solid State Commun. 71, 527 (1989).

${ }^{16}$ F. Stern, Phys. Rev. Lett. 18, 546 (1967).

${ }^{17}$ R. Ritchie, Phys. Rev. 106, 874 (1957).

${ }^{18}$ J. Rogers III, P. Cutler, T. Feuchtwang, N. Miskovsky, and A. Lucas, Surf. Sci. 141, 61 (1984).

${ }^{19}$ J. Rogers III, P. Cutler, T. Feuchtwang, N. Miskovsky, and A. Lucas, Surf. Sci. 181, 436 (1986).

${ }^{20} \mathrm{H}$. Ehrenreich and M. Cohen, Phys. Rev. 115, 786 (1959).

${ }^{21}$ S. Adler, Phys. Rev. 126, 413 (1962).

${ }^{22}$ D. Dahl and L. Sham, Phys. Rev. B 16, 651 (1977).

${ }^{23}$ A. Rajagopal, Phys. Rev. B 15, 4264 (1977).

${ }^{24}$ J. Devreese, F. Brosens, and L. Lemmens, Phys. Rev. B 21, 1349 (1980).

25J. Dryzek and A. Czapla, Phys. Rev. Lett. 58, 721 (1986).

${ }^{26}$ A. Kamgar, P. Kneschaurek, G. Dorda, and J. Koch, Phys. Rev. Lett. 32, 1251 (1974).

${ }^{27}$ P. Kneschaurek, A. Kamgar, and J. Koch, Phys. Rev. B 14, 1610 (1976). 


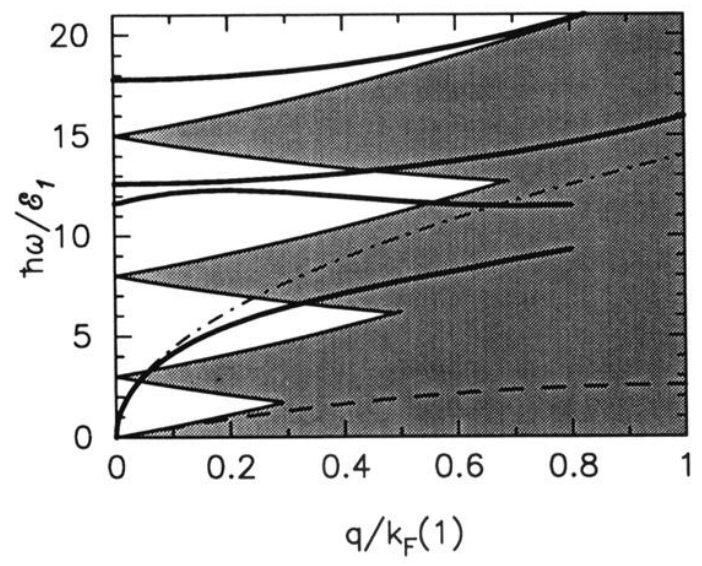

FIG. 3. The zero-point inventory of the dielectric determinant for one occupied subband (solid curves). The electronhole continuum is marked and shaded, also Stern's dispersion relation (dashed-dotted) is drawn; $E_{F} / \mathscr{E}_{1}=3.5, n^{(3 \mathrm{D})}=5 \times 10^{20}$ $\mathrm{cm}^{-3}, \gamma=0$. 

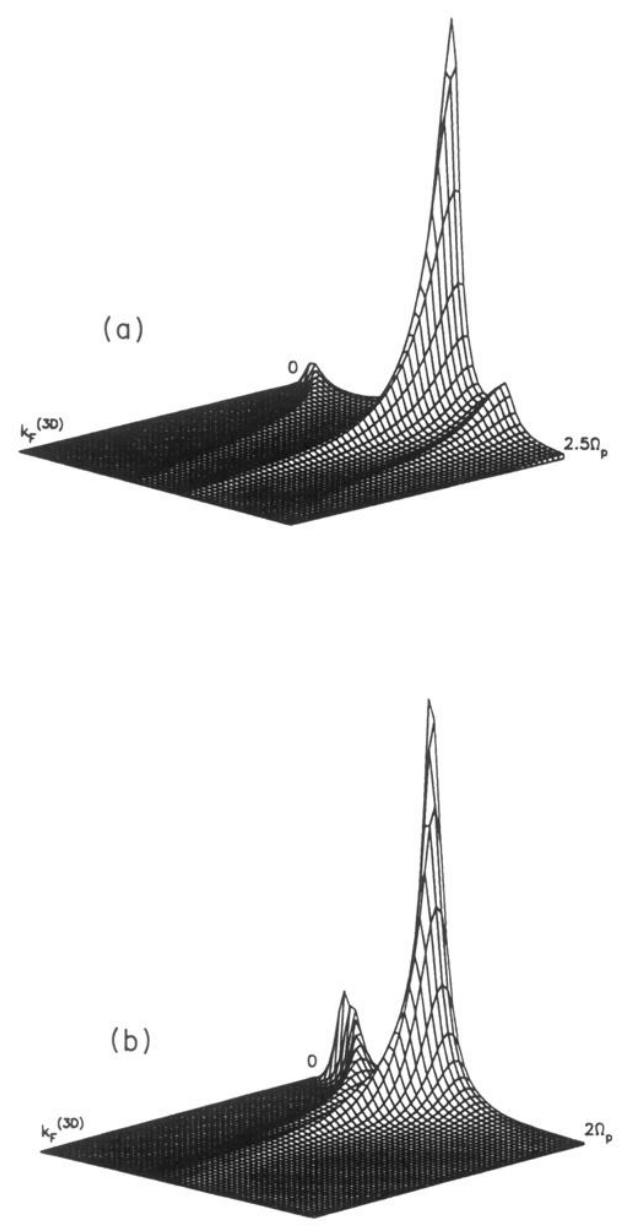

FIG. 5. The absorption function $\mathcal{P}(\omega, q)$ for (a) $l_{F}=1$, $E_{F} / \mathscr{E}_{1}=2.1$ and (b) $l_{F}=4, E_{F} / \mathscr{E}_{1}=17.6$. We used a damping of $\gamma=0.1 \Omega_{p}$. 